\title{
An Italian multicenter retrospective-prospective observational study on neurological manifestations of COVID-19 (NEUROCOVID)
}

\author{
Carlo Ferrarese ${ }^{1}$ (D) Vincenzo Silani ${ }^{2} \cdot$ Alberto Priori $^{3} \cdot$ Stefania Galimberti $^{4} \cdot$ Elio Agostoni $^{5} \cdot$ Salvatore Monaco $^{6}$. \\ Alessandro Padovani ${ }^{7} \cdot$ Gioacchino Tedeschi $^{8} \cdot$ on behalf of Italian Society of Neurology (SIN)
}

Received: 2 May 2020 / Accepted: 5 May 2020 / Published online: 19 May 2020

(C) Fondazione Società Italiana di Neurologia 2020

\begin{abstract}
Neurological manifestations of COVID-19 have been described in both single case reports and retrospective scanty case series. They may be linked to the potential neurotropism of the SARS-COV-2 virus, as previously demonstrated for other coronaviruses. We report here the description of a multicenter retrospective-prospective observational study promoted by the Italian Society of Neurology (SIN), involving the Italian Neurological Departments, who will consecutively recruit patients with neurological symptoms and/or signs, occurred at the onset or as a complication of COVID-19. Hospitalized patients will be recruited either in neurological wards or in COVID wards; in the latter cases, they will be referred from other specialists to participant neurologists. Outpatients with clinical signs of COVID and neurological manifestations will be also referred to participating neurologists from primary care physicians. A comprehensive data collection, in the form of electronic case report form (eCRF), will register all possible neurological manifestations involving central nervous systems, peripheral nerves, and muscles, together with clinical, laboratory (including cerebrospinal fluid, if available), imaging, neurological, neurophysiological, and neuropsychological data. A follow-up at hospital discharge (in hospitalized patients), and for all patients after 3 and 6 months, is also planned. We believe that this study may help to intercept the full spectrum of neurological manifestations of COVID-19 and, given the large diffusion at national level, can provide a large cohort of patients available for future more focused investigations. Similar observational studies might also be proposed at international level to better define the neurological involvement of COVID-19.
\end{abstract}

Keywords COVID-19 $\cdot$ Neurological manifestations $\cdot$ NEUROCOVID $\cdot$ Observational study

\section{Introduction}

In the recent outbreak of COVID-19 pandemic, emerging in China and then exported first to Iran and Italy, particularly in

Carlo Ferrarese

carlo.ferrarese@unimib.it

1 Department of Medicine and Surgery and Milan Center for Neuroscience (NeuroMI), University of Milano-Bicocca and San Gerardo Hospital, ASST-Monza, Via Pergolesi 33, Monza, Italy

2 Istituto Auxologico Italiano, IRCCS, Department of Neurology and Laboratory of Neuroscience - Department of Pathophysiology and Transplantation, "Dino Ferrari" Center, Università degli Studi di Milano, Milan, Italy

3 Clinica Neurologica III, "Centro Aldo Ravelli” per le Terapie Neurologiche Sperimentali, Dipartimento di Scienze della Salute, Polo Universitario San Paolo, Università degli Studi di Milano, ASST Santi Paolo e Carlo, Milano, Italy the northern Lombardy region, and thereafter to a growing number of European countries and America, single case reports of co-occurrence of neurological disorders are being reported $[1,2]$. A recent self-reported questionnaire on 59

4 Bicocca Bioinformatics Biostatistics and Bioimaging Centre, Department of Medicine and Surgery, University of Milano Bicocca, Monza, Italy

5 S.C. Neurologia e Stroke Unit ASST Grande Ospedale Metropolitano Niguarda, Milan, Italy

6 Department of Neuroscience, Biomedicine and Movement Sciences, University of Verona, Verona, Italy

7 Department of Neurology, ASST Spedali Civili and University of Brescia, Brescia, Italy

8 Department of Advanced Medical and Surgical Sciences, University of Campania “L. Vanvitelli", Naples, Italy 
COVID hospitalized patients in Milan revealed a $20 \%$ prevalence of olfactory and taste disorders [3]. A retrospective observational study by Mao et al. [4] collected, from 16 January 2020 to 19 February 2020, two hundred fourteen hospitalized patients with severe COVID infection in three hospitals of Wuhan, China. Seventy-eight (36.4\%) patients had neurologic manifestations. More severe patients were likely to have neurologic symptoms, such as acute cerebrovascular diseases, impaired consciousness, and skeletal muscle injury. At present, this is the first and only report on detailed neurologic manifestations of the hospitalized patients with COVID-19, and authors observed that involvement of the nervous system carried a poor prognosis. Therefore, they called physicians to pay close attention to any neurologic manifestations, in addition to the symptoms of respiratory system. Furthermore, on March 4, 2020, Beijing Ditan Hospital reported for the first time a case of viral encephalitis and the researchers confirmed the presence of SARS-CoV-2 in the cerebrospinal fluid by genome sequencing [5]. Autopsy reports in some deceased patients have revealed brain tissue edema and partial neuronal degeneration [6]. A further series of patients has been recently reported from Strasbourg, France: agitation and corticospinal tract signs were reported in $69 \%$ and $67 \%$ of the patients, respectively [7].

Indeed, SARS-CoV-2, as previously demonstrated for other coronaviruses [8-10], may infect nervous system, skeletal muscle, and respiratory tract, since all systems share the same ACE-2 receptors present in respiratory system [11]. It has been also hypothesized that the potential neuroinvasion of SARS-CoV2 is partially responsible for the acute respiratory failure of patients with COVID19 [12]. Stroke patients with COVID may have a worse prognosis, for many reasons (delays in transports to hospitals and in diagnostic procedures with decreased thrombolytic and thrombectomy treatments, lack of intensivecare units, proinflammatory, and prothrombotic status). In addition, critically ill patients with severe SARS-CoV-2 infections often show elevated levels of D-dimer and severe platelet reduction, which may render them prone to acute cerebrovascular events [13].

Moreover, many neurological diseases (multiple sclerosis, Guillain-Barré, and chronic inflammatory demyelinating polyneuropathy [CIDP], myasthenia) have immunological pathogenesis and might be exacerbated or triggered by COVID.

For all these reasons, close monitoring of any possible neurological manifestation at the onset, during or after COVID19 , is mandatory for the neurologic community.

We propose here a national multicenter retrospectiveprospective study, opened to Italian neurological departments and sponsored by the Italian Society of Neurology (SIN), to investigate the occurrence of neurological manifestations in patients with COVID-19. Since most infected patients, who may also present neurological complications, are not hospitalized, but followed by primary care physician, they will be asked to refer such patients to participating neurologists.

\section{Study objective}

The primary aim of the study is to collect detailed information on any neurological manifestation of COVID-19. More specifically, the aims are to gather data on the following: (1) the appearance of neurologic symptoms and/or signs at COVID19 onset or during the disease course, (2) the exams performed for the diagnosis of the neurological involvement, (3) the clinical course of both the COVID-19 infection and the neurological events, but also the occurrence of possible long-term neurological complications within a 6-month period of follow-up.

\section{Design}

For this aim, we propose a multicenter observational retrospective-prospective study, performed at national level under the patronage of the Italian Society of Neurology. The enrolment will be both at hospital and community levels. The neurologic participating centers will recruit consecutive COVID-19 patients followed directly in the neurological wards or referred from COVID wards of the same hospital with any neurological manifestation occurring at the onset, during, or after recovery from infection. Since in Italy (as well as in many countries) most COVID-19 patients are followed at home by primary care physicians, they will be asked from participating centers to refer them all COVID-19 patients with possible neurologic manifestations. The duration of the recruiting period will be 6 months, starting from March 1, 2020, to August 31, 2020, with a mixture of retrospective and prospective data collection, and the follow-up period will be of 6 months (until February 28, 2021).

\section{Participants}

All participants will be aged 18 or over and will fulfil one of the two following criteria:

1. Positive PCR test for SARS-CoV-2 RNA on nasopharyngeal swab and/or positive serological test and/or positive chest CT for interstitial pneumonia due to COVID-19, independently from clinical severity, if hospitalized, and appearance of neurological symptoms or signs

2. Clinical picture suggestive of COVID-19 (fever above $37.5^{\circ} \mathrm{C}$, symptoms of respiratory system infection) and appearance of neurological symptoms or signs for subjects referred from primary care physician.

The prospectively included participants will provide a written informed consent to study participation. The study has been approved by the Ethics Committee of the Promoter 
(Istituto Auxologico Italiano, IRCCS Milan; 16 April 2020) and will be approved in all the participating centers.

\section{Data collection}

To describe any possible neurological manifestation of COVID-19, its temporal relation with the onset of systemic infection and its evolution in time, a comprehensive data collection, has been designed that includes demographic, lifestyle habits, comorbidities, concomitant therapies, clinical characteristics, therapies and outcomes of the COVID-19 infection, clinical, imaging, biochemical, and neurophysiological data of the neurological manifestations of COVID-19. All data will be securely stored in an electronic case report form (eCRF) developed with the REDCap Cloud platform. Data access will be protected by username and password according to different profiles, depending on the user. In particular, centers will have access only to data relative to their own center, whereas the data manager and the biostatisticians will have online access to the entire database. The data coordinating center will be at the University of Milano-Bicocca.

\section{Sample size}

No formal sample size calculation has been performed for this study. However, we expect to recruit a minimum of 1000 hospitalized patients, considering that we hope to involve a large number of centers in the Northern Italian regions where the epidemic is much more spread. We cannot anticipate the amount of non-hospitalized patients since this component will depend on the sensibility of the primary care physicians.

\section{Statistical analysis}

Continuous variables will be described with median and interquartile range (IQR), or mean and standard deviation (SD), as appropriate, and categorical data will be reported as absolute and relative frequencies.

The descriptive analysis will allow the clinical picture of the COVID-19 patients involved in the study, the characterization, and quantification of the degree of their neurological involvement at diagnosis, during the course of the disease or in the subsequent period. The estimate of the prevalence of neurological manifestations and the estimate of the risk of developing them will be calculated overall and for specific disorders, along with the corresponding $95 \%$ confidence intervals (using the total of COVID-19 patients in the centers involved in the study). Data on cases referred from primary care physicians will be used to confirm the spectrum of neurologic manifestations and will be eventually analyzed in case data from administrative registries will be available. Analyses will be performed with the use of SAS software (version 9.4) and R software (version 3.6).

\section{Monitoring of the trial}

A data manager, under the supervision of the biostatistician, will perform periodic quality checks in terms of completeness, consistence, and coherence of data. Reports with queries center-specific will be prepared.

\section{Discussion}

As the number of patients with COVID-19 increases worldwide, neurological manifestations have been progressively described mostly in preliminary reports and in retrospective case series of patients. However, so far, no prospective study has been designed to intercept any possible neurologic manifestation occurring in COVID patients, either in severe hospitalized patients, or in mild or asymptomatic patients who, in Italy, are invited to stay at home. The main aim of this retrospective-prospective observational study, therefore, is to collect the maximum number of patients with neurologic manifestations of COVID-19, to describe their temporal relation with COVID-19 symptomatology and to follow them for up to 6 months. Indeed, neurologists need to pay close attention to COVID-19 neurological manifestations, which may have contributed to their demise, especially for those with severe infections. Furthermore, when seeing patients with neurological manifestations in this pandemic period, neurologists should consider COVID-19 infection as a possible concomitant pathology, so to avoid delayed diagnosis or misdiagnosis and prevention of transmission.

To reach this goal, due to the particularly severe pandemic expression in Italy and specifically in Norther Italy, a comprehensive data collection, in the form of eCRF, has been rapidly designed to register all possible neurological manifestations involving central nervous systems, peripheral nerves and muscles, together with clinical, laboratory (including cerebrospinal fluid, if available), imaging, neurological, neurophysiological, and neuropsychological data. The eCRF we provide is quite accurate to detect also mild neurological symptoms such as hypogeusia and hyposmia, both seeming to be particularly represented also in early stage of the disease, representing a possible clinical screening tool to further orientate testing of paucisymptomatic individuals [2]. A large part of Italian patients is followed at home by primary care physicians, for this reason, we also planned to recruit these patients, referred by primary care physicians to participating neurologists.

A follow-up at hospital discharge (in hospitalized patients), and for all patients after 3 and 6 months, has been also designed, to provide the first analysis on the natural history of COVID-related neurological manifestations. 
The main goal of this study is to rapidly define the patients suffering of nervous and muscles impairment and further make aware the neurological community of the different aspects of neuroCOVID-19 that need to be fully considered for further studies. The main limitation of the previous studies is the limited cases considered, with biases in clinical observations and data interpretation. It would be better, at this point, to avoid collection of data from small case series and plan multicenter studies, to include a large number of patients on a national basis, under the auspices of the Italian Society of Neurology (SIN). This preliminary analysis will represent a useful referral for both the neurological scientific community and the authorities, to further dissect the different aspects of the neuroCOVID-19, addressing further researches in areas of specific interest. Close monitoring of the neurological manifestations of COVID-19 may also provide indications for specific diagnostic and/ or therapeutic procedures of unpredictable benefit in subsets of COVID-19 patients with various central or peripheral neurological involvement.

We believe that this Italian multicentric observational study will rapidly provide, using adequate $\mathrm{eCRF}$, accurate information related to the central, peripheral, and muscle involvement during the COVID-19 pandemic in the large national population of Italy. The study may also largely contribute in shedding light on the pathogenesis of the neurological complications in COVID-19 that is probably complex, involving a currently unknown set of interactions between viral properties, tissue susceptibility, and host immune response even in the nervous system. The study may also add in recognizing that some patients that recover from COVID-19 may complain of lasting neurological sequelae that may have been overlooked at the time of acute illness. Therefore, long-term follow-up for neurological deficits may be considered as essential in Italy and the rest of the world. Furthermore, the gained sensitivity of the new generations of neurologists in the residency programs will be a contributing factor in further defining the multiple neuroclinical features of COVID-19 [14].

Possible collaborations with EAN and with affiliated European National Neurological Societies, as well as with AAN and other Neurological Societies worldwide, are welcome and might reveal country or region-specific patterns of the neurological manifestations of COVID-19.

Acknowledgments We thank Dr. Paola Alberti, MD, $\mathrm{PhD}$, for editorial assistance.

\section{Compliance with ethical standards}

Conflict of interest $\mathrm{CF}$ is in the Editorial Board of Parkinson Disease and Neurological Sciences; Coordinator of PRIN 2017, Grant: 2017CY3J3W, received grants and compensations for consulting services from Biogen, DOCPharma, Merck, Roche.
VS is in the Editorial Board of Amyotroph Lateral Sclerosis, European Neurology, American Journal of Neurodegenerative Diseases, Frontiers in Neurology; received compensation for consulting services and/or speaking activities from AveXis, Cytokinetics, Italfarmaco, and Zambon; and receives or has received research supports from the Italian Minsitry of Health (Grant RF-201302355764), Fondazione Italiana di Ricerca per la SLA - AriSLA (Grants exomafals and Novals), Fondazione Regionale per la Ricerca Biomedica regione Lombarda (Project nr. 2015-0023), and E-RARE JTC 2018 (Project Repetomics). $\mathrm{APr}, \mathrm{APa}, \mathrm{EA}, \mathrm{GT}, \mathrm{SG}$, and SM have nothing to disclose.

Ethical approval Approved by Ethic Committe of Auxologic Institute, Milan, on April 16, 2020.

\section{References}

1. Alberti P, Beretta S, Piatti M, Karantzoulis A, Piatti ML, Santoro P, Viiganò M, Giovannelli G, Pirro F, Montisano DA, Appollonio I, Ferrarese C (2020) Guillain-Barré syndrome related to COVID-19 infection, Neurology. Neuroimmunol Neuroinflamm 7(4)e741

2. Filatov A, Sharma P, Hindi F, Espinosa PS (2020) Neurological complications of coronavirus disease (COVID-19): encephalopathy. Cureus 12(3):e7352

3. Giacomelli A, Pezzati L, Conti F, Bernacchia D, Siano M, Oreni L, Rusconi S, Gervasoni C, Ridolfo AL, Rizzardini G, Antinori S, Galli M (2020) Self-reported olfactory and taste disorders in SARS-CoV-2 patients: a cross-sectional study. Clin Infect Dis pii: ciaa330. doi: https://doi.org/10.1093/cid/ciaa330

4. Mao L, Jin H, Wang M, Hu Y, Chen S, He Q, Chang J, Hong C, Zhou Y, Wang D, Miao X, Li Y, Hu B (2020) Neurologic manifestations of hospitalized patients with coronavirus disease 2019 in Wuhan, China. JAMA Neurol doi: https://doi.org/10.1001/ jamaneurol.2020.1127

5. Wu Y, Xu X, Chen Z, Duan J, Hashimoto K, Yang L, Liu C, Yang C (2020) Nervous system involvement after infection with COVID19 and other coronaviruses. Brain Behav Immun pii: S08891591(20)30357-3. doi: https://doi.org/10.1016/j.bbi.2020.03.031

6. Li Z, Huang Y, Guo X (2020) The brain, another potential target organ, needs early protection from SARS-CoV-2 neuroinvasion. Sci China Life Sci 63(5):771-773. doi: https://doi.org/10.1007/ s11427-020-1690-y

7. Helms J, Kremer S, Merdji H, Clere-Jehl R, Schenck M, Kummerlen C, Collange O, Boulay C, Fafi-Kremer S, Ohana M, Anheim M, Meziani F (2020) Neurologic features in severe SARSCoV-2 infection. N Engl J Med doi: https://doi.org/10.1056/ NEJMc2008597

8. Hung EC, Chim SS, Chan PK, Tong YK, Ng EK, Chiu RW, Leung CB, Sung JJ, Tam JS, Lo YM (2003) Detection of SARS coronavirus RNA in the cerebrospinal fluid of a patient with severe acute respiratory syndrome. Clin Chem. 49(12):2108-2109

9. Lau KK, Yu WC, Chu CM, Lau ST, Sheng B, Yuen KY (2004) Possible central nervous system infection by SARS coronavirus. Emerg Infect Dis. 10(2):342-344

10. Desforges M, Le Coupanec A, Dubeau P, Bourgouin A, Lajoie L, Dubé M, Talbot PJ (2019) Human coronaviruses and other respiratory viruses: underestimated opportunistic pathogens of the central nervous system? Viruses 12(1) pii: E14. doi: https://doi.org/10. 3390/v12010014

11. Baig AM (2020) Neurological manifestations in COVID-19 caused by SARS-CoV-2. CNS Neurosci Ther 26(5):499-501. doi: https:// doi.org/10.1111/cns.13372

12. Li YC, Bai WZ, Hashikawa T (2020) The neuroinvasive potential of SARS-CoV2 may play a role in the respiratory failure of 
COVID-19 patients. J Med Virol doi: https://doi.org/10.1002/jmv. 25728

13. Wang D, Hu B, Hu C, Zhu F, Liu X, Zhang J, Wang B, Xiang H, Cheng Z, Xiong Y, Zhao Y, Li Y, Wang X, Peng Z (2020) Clinical characteristics of 138 hospitalized patients with 2019 novel coronavirus-infected pneumonia in Wuhan, China. JAMA doi: https://doi.org/10.1001/jama.2020.1585
14. Calcagno N, Colombo E, Maranzano A, Pasquini J, Keller Sarmiento IJ, Trogu F, Silani V (2020) Rising evidence for neurological involvement in COVID-19 pandemic. Neurol Sci. In press doi: https://doi.org/10.1007/s10072-020-04447-w

Publisher's note Springer Nature remains neutral with regard to jurisdictional claims in published maps and institutional affiliations. 